\title{
My palliative care rotation: Lessons of gratitude, mindfulness, and kindness
}

\section{Muhammad Wajahat, MBBS}

s a psychiatry resident and as a part of consultation-liaison service, I have visited many palliative care patients to assist other physicians in managing psychiatric issues such as depression, anxiety, or delirium. But recently, as the first resident from our Department of Psychiatry who was sent to a palliative care rotation, I followed these patients as a part of a primary palliative care team. Doing so allowed me to see patients from the other side of the bridge.

Palliative care focuses on providing relief from the suffering and stress of a patient's illness, with the primary goal of improving the quality of life of the patient and their families. The palliative care team works in collaboration with the patient's other clinicians to provide an extra layer of support. They provide biopsychosociocultural interventions that are in harmony with the needs of the patient rather than the prognosis of the illness. To do so, they first must evaluate the needs of the patient and their family. This is a time-consuming, energyconsuming, emotionally draining job.

During my palliative care rotation, I attended table rounds, bedside rounds, family meetings, long counseling sessions, and disposition planning meetings. This rotation also gave me the opportunity to place my feet in the shoes of a palliative care team and to reflect on how it feels to be the physician of a patient who is dying, which as a psychiatric resident I had seldom experienced. I learned that although working with patients who are dying can cause stress, burnout, and compassion fatigue, it

also helps physicians appreciate the little things in life. To appreciate all the blessings we have that we usually take for granted. To practice gratitude. To be kind.

Upon reflection, I learned that the rounds of palliative care are actually mindfulnessbased discussions that provide cushions of supportive work, facilitate feelings of being in control, tend to alleviate physical as well as mental suffering, foster clear-sighted hope, and assist in establishing small, subjectively significant, realistic goals for the patient's immediate future, and to help the patient achieve these goals.

\section{A valuable lesson from a patient}

I want to highlight a case of a 65-year-old woman I first visited while I was shadowing my attending, who had been providing palliative care to the patient and her family for several months. The patient was admitted to a tertiary care hospital because cancer had invaded her small bowel and caused mechanical obstruction, resulting in intractable vomiting, abdominal distension, and anorexia. She underwent open laparotomy and ileostomy for symptomatic relief. A nasogastric tube was placed, and she was put on total parenteral nutrition. The day

LET YOUR VOICE BE HEARD

CURRENT PSYCHIATRY invites psychiatry residents to share their views on professional or clinical topics for publication in Residents' Voices. E-mail jbauer@mdedge.com for author guidelines.

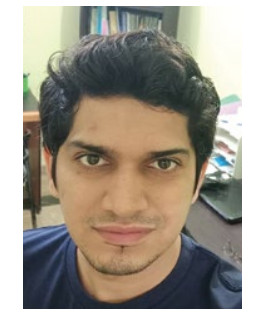

Dr. Wajahat is Chief Resident, PGY-4, Department of Psychiatry, Aga Khan University Hospital, Karachi, Pakistan.

\section{Disclosure}

The author reports no financial relationships with any companies whose products are mentioned in this article, or with manufacturers of competing products.

doi: 10.12788/cp.0165 


\section{Clinical Point}

\section{My palliative care rotation gave me the opportunity to reflect on how it feels to be the physician of a patient who is dying}

I met her was her third postoperative day. She had been improving significantly, and she wanted to eat. She was missing food. Most of the discussion in the round among my attending, the patient, and her family was centered around how to get to the point where she would be able to eat again and appreciate the taste of biryani.

What my attending did was incredible. After assessing the patient's needs, he instilled a realistic hope: the hope of tasting food again. The attending, while acknowledging the patient's apprehensions, respectfully and supportively kept her from wandering into the future, made every possible attempt to bring her attention back to the present moment, and helped her establish goals for the present and her immediate future. My attending was not toxic-positive, forcing his patient to uselessly revisit her current trauma.
Instead, he was kind, empathic, and considerate. His primary focus was to understand rather than to be understood, to help her find meaning, and to improve her quality of life-a quality she defined for herself, which was to taste the food of her choice.

That day, when I returned to my working station in the psychiatry ward and had lunch in the break room, I thought, "When I eat, how often do I think about eating?" Mostly I either think about work, tasks, and presentations, or I scroll on social media.

Our taste buds indeed get adapted to repetitive stimulation, but the experience of eating our favorite dish is the naked truth of being alive, and is something that I have been taking for granted for a long time. These are little things in life that I need to appreciate, and learn to cultivate their power. 